\title{
Significance of Serum Expression of Rho Kinase, Endothelin-1, and Nitric Oxide in Pediatric Patients With Left-to-right Shunt Congenital Heart Disease Accompanied by Pulmonary Hypertension
}

\author{
Xing-Zhen Sun \\ Huai'an First Paople"s Hospital \\ Jian Hu \\ Huai'an First People's Hospital \\ Xiang-Yang Tian ( $\nabla$ tianxy_edu@163.com ) \\ Huai'an First People's Hospital \\ Ze Hong \\ Huai'an First People's Hospital
}

Research article

Keywords: Congenital heart disease, pulmonary hypertension, Rho kinase, endothelin-1, nitric oxide

Posted Date: July 20th, 2020

DOI: https://doi.org/10.21203/rs.3.rs-42404/v1

License: (a) (i) This work is licensed under a Creative Commons Attribution 4.0 International License.

Read Full License 


\section{Abstract \\ Background}

The study aimed to investigate the serum expression levels of Rho kinase, endothelin-1 (ET-1), and nitric oxide (NO) in pediatric patients with left-to-right shunt congenital heart disease (CHD) and their correlation with pulmonary artery systolic pressure (PASP).

\section{Methods}

90 pediatric patients diagnosed with left-to-right shunt CHD and hospitalized were selected as the study objects. They were divided into three groups according to their PASP: the non-pulmonary artery hypertension (non-PAH) group (PASP $<30 \mathrm{mmhg}$ ), the mild PAH group (PASP $30-49 \mathrm{mmhg}$ ), and the moderate to severe PAH group (PASP $\geq 50 \mathrm{mmhg}$ ). Healthy children were selected as the control group. Enzyme-linked immunosorbent assay, radioimmunoassay, and nitrate reductase assay were used to determine the patients' serum levels of Rho kinase, ET-1, and NO, respectively. The correlation between PASP and the above indexes was analyzed.

\section{Results}

In pediatric patients with left-to-right shut $\mathrm{CHD}$, the serum expression levels of Rho kinase and ET-1 were higher than in the control group members, while the serum expression level of NO was lower. In pediatric patients with $\mathrm{CHD}$ accompanied by pulmonary hypertension $(\mathrm{PH})$, the expression levels of Rho kinase and ET-1 were even higher, and the level of NO even lower, than in patients and control group members without PH. The serum levels of Rho kinase and ET-1 were positively correlated with PASP, while the serum level of NO was negatively correlated with PASP.

\section{Conclusion}

Rho kinase, ET-1, and NO may be involved in the development of PH in pediatric patients with left-to-right shunt CHD.

\section{Background}

Pulmonary hypertension $(\mathrm{PH})$ is the most common and most serious complication of left-to-right shunt congenital heart disease (CHD). It is characterized by increased reactivity of pulmonary vasoconstriction and by pulmonary vascular structural reconstruction. The mechanism of the occurrence and regulation of $\mathrm{PH}$ remains unclear ${ }^{[1,2]}$. Recent studies have shown that Fasudil, a selective inhibitor of the Rho kinase signaling pathway, can significantly inhibit the progress of pulmonary vasoconstriction and pulmonary 
vascular structural reconstruction in experimental animals with $\mathrm{PH},{ }^{[3]}$ and it can improve the balance between endothelin-1 (ET-1) and nitric oxide (NO) by inhibiting the synthesis and secretion of ET-1 ${ }^{[4]}$ and promoting the synthesis and secretion of $\mathrm{NO}^{[5]}$ in endothelial cells, thereby enhancing endotheliummediated vascular relaxation. This suggests that Rho kinase, ET-1, and NO may play an important role in the development of PH. The present study aimed to investigate the role of Rho kinase, ET-1, and NO in the pathogenesis of $\mathrm{CHD}$ accompanied by $\mathrm{PH}$ by observing the differences in their serum expression levels in pediatric patients with $\mathrm{CHD}$ and their correlation with pulmonary artery pressure.

\section{Methods}

\section{- Materials and methods}

- General data

Between June 2015 and September 2016, 90 pediatric patients with left-to-right shunt CHD hospitalized in the Departments of Pediatrics and Cardiology at Huai'an First Hospital Affiliated to Nanjing Medical University were enrolled in the study. The inclusion criteria were as follows: (1) patients in accordance with the diagnostic criteria of left-to-right shunt $\mathrm{CHD},{ }^{[6]}$ and (2) patients with complete clinical data. The exclusion criteria were as follows: (1) patients with cardiac infection or cardiac failure, (2) patients with other severe complications, and (3) patients with incomplete clinical data. There were 48 males and 42 females between 2 months and 12 years of age, with a median age of 4.2 years. The pediatric patients with CHD were divided into three group based on their pulmonary artery systolic pressure (PASP) measured at resting state by Doppler echocardiography: the non-pulmonary artery hypertension (nonPAH) group (PASP <30 mmhg, 30 cases), the mild PAH group (PASP $30-49 \mathrm{mmhg}, 30$ cases), and the moderate to severe PAH group (PASP $\geq 50 \mathrm{mmhg}, 30$ cases). Healthy children receiving physical examinations in the outpatient clinic during the same period were selected as the healthy normal control group, which included 18 males and 12 females between 2 months and 12 years of age (median age: 4.5 years). There was no significant difference in age, gender, or other general data in children in each group (P> 0.05 in each), and the data were comparable. The content of the present study was approved by the Medical Ethics Committee (Ethics approval number: IRB-KPJ2016-010-01) and written informed consent was obtained from the parents of all children who participated in the study.

\subsection{Methods}

\subsubsection{Detection of pulmonary artery pressure by Doppler echocardiography ${ }^{[7]}$}

According to the measurement of tricuspid regurgitation pressure difference by the Doppler echocardiography under resting state, the right ventricular inflow tract was taken as the standard section and maximum tricuspid regurgitation velocity $(\mathrm{V}, \mathrm{m} / \mathrm{s})$ was measured by continuous Doppler echocardiography under a resting conditions. The tricuspid regurgitation pressure difference ( $\mathrm{PG}, \mathrm{mmHg})$ was calculated using the simplified Bernoulli equation: $P G=4 V 2$. PASP was determined by adding right 
atrial pressure (RAP) to PG; RAP was generally set to $10 \mathrm{mmHg}$. PASP is therefore equivalent to PG + RAP $=4 \mathrm{~V} 2+10$.

\subsubsection{Sample collection and assay}

A $2 \mathrm{ml}$ sample of venous blood was drawn from each child in the morning under fasting conditions and placed in a tube containing $30 \mu \mathrm{M}$ of Ethylenediamine tetraacetic acid disodium salt and $40 \mu \mathrm{M}$ of aprotinin. The sample was centrifuged at $3000 \mathrm{r} / \mathrm{min}$ at a radius of $12 \mathrm{~cm}$ for $10 \mathrm{~min}$. The supernatant was removed and stored for further analysis in a refrigerator at $-20^{\circ} \mathrm{C}$. An additional $4 \mathrm{ml}$ of blood drawn from each child was put in an empty test tube without anticoagulant and then centrifuged, and the supernatant was removed and refrigerated for further analysis. The serum levels of Rho kinase, ET-1, and NO in each group were detected using enzyme-linked immunosorbent assay (ELISA kit provided by R\&D Systems of the United States), radioimmunoassay (ET-1 kit provided by the radioimmunoassay institute of science and technology development center of the PLA General Hospital), and nitrate reductase assay (NO kit provided by Nanjing Jiancheng Bioengineering Research Institute), respectively. All tests were carried out by a specially assigned person in strict accordance with the kit instructions.

\subsection{Statistical analysis}

SPSS 16.0 software was used for statistical analysis of the data. The data were expressed as mean \pm standard deviation ( $\overline{\mathrm{x}} \pm \mathrm{S}$ ). An ANOVA and a SNK-qtest were performed on the data from each group, followed by Pearson correlation analysis. $P<0.05$ was considered statistically significant.

\section{Results}

2.1 The serum levels of Rho kinase, ET-1 and NO in each group

The results of the study are shown in Table 1. To summarize, the serum levels of Rho kinase and ET-1 in pediatric patients with CHD were significantly higher than those of patients in the control group $(P<0.01)$, and the serum levels of patients in the mild PAH and moderate to severe PAH groups were also significantly higher than those of patients in the non-PAH group and the control group $(P<0.01)$. The serum level of $\mathrm{NO}$ in pediatric patients with $\mathrm{CHD}$ was significantly lower than that of the control group $(P<$ $0.01)$, and the levels in the mild PAH and moderate to severe PAH groups were also significantly lower than in the non-PAH and control groups $(P<0.01)$.

- Correlation between the serum levels of Rho kinase, ET-1, NO and PASP

Pearson correlation analysis showed that the serum levels of Rho kinase and ET-1 were significantly and positively correlated with PASP ( $r=0.707$ and 0.888 , respectively; $P<0.01$ in both), while the level of NO was negatively correlated with PASP $(r=-0.696, P<0.01)$. There was a significantly negative correlation between the serum level of NO and the levels of Rho kinase and ET-1 $(r=-0.522,-0.592$, respectively; $P<$ 0.01 in both). (Figure. $1 \mathrm{~A}-\mathrm{E})$. 


\section{Discussion}

$\mathrm{PH}$ caused by increased pulmonary blood flow is the most common complication of $\mathrm{CHD}$ with left-to-right shunt. The severity of $\mathrm{PH}$ directly affects the surgical effect and prognosis of patients ${ }^{[8]}$. At present, there is no complete explanation of the mechanism of occurrence and regulation of $\mathrm{PH}$ in $\mathrm{CHD}$, butit has been suggested that $\mathrm{PH}$ is caused by many factors, such as the proliferation of pulmonary vascular smooth muscle cells, the increase of extracellular matrix components, the myostratification of non-muscular arteries, the swelling and hypertrophy of endothelial cells, and, finally, the thickening of the pulmonary artery wall and stenosis of the lumen of the pulmonary artery ${ }^{[9]}$.

The role of the Rho-kinase signaling pathway in the pathogenesis of $\mathrm{PH}$ has drawn increasing attention in recent years ${ }^{[10,11]}$. In particular, Fasudil, a selective inhibitor of the Rho kinase signaling pathway, has been shown to significantly inhibit the progress of pulmonary vasoconstriction and pulmonary vascular structural reconstruction in experimental animals with $\mathrm{PH}$. Thus, it is postulated that the abnormal activation of Rho kinase plays an important role in the pathogenesis of $\mathrm{PH}$ by directly affecting the contraction of smooth muscle cells, changing the balance between endothelium-derived relaxing factors and contraction factors, and regulating the expression of cell growth genes and other mechanisms ${ }^{[12]}$. Recent studies have shown that Rho kinase can be activated by a variety of upstream stimulation signals $[4,5,12]$, including ET-1, NO, angiotensin II (Angll), and oxidative stress. While previous studies have shown that all of these stimulating factors can cause PH and structural remodeling, ET- 1 is by the far the strongest vasoconstrictor and a powerful mitogenic agent of smooth muscle cells. An increase in ET-1 can not only strongly contract the pulmonary artery but also promote the proliferation of vascular smooth muscle and fibrous tissue, increase pulmonary vascular resistance, and participate in the reconstruction of pulmonary vessels ${ }^{[12,13]}$. NO can also play an important role: As an endothelium-derived relaxing factor, NO can selectively relax the pulmonary artery, inhibit the adhesion and aggregation of platelets, and reduce pulmonary artery pressure $[5,10,14]$.

The present study investigated the differences in serum levels of Rho kinase, ET-1, and NO in pediatric patients with CHD accompanied by PH. The serum levels of Rho kinase, ET-1, and NO in pediatric patients with $\mathrm{CHD}$ accompanied by $\mathrm{PH}$ were compared to those of healthy children with normal pulmonary artery pressure. It was found that the levels of Rho kinase and ET-1 were significantly higher in pediatric patients with $\mathrm{CHD}$ than they were in the healthy control group $(P<0.01)$, and the serum levels of patients in the mild PAH and moderate to severe PAH groups were also significantly higher than those of patients in the non-PAH and control groups $(P<0.01)$. The serum level of NO in pediatric patients with CHD was significantly lower than in the healthy control group $(P<0.01)$, and the serum level of NO in the mild PAH and moderate to severe PAH groups was significantly lower than in the non-PAH and healthy control groups $(P<0.01)$. The serum levels of Rho kinase and ET-1 were significantly and positively correlated with PASP ( $r=0.707$ and 0.888 , respectively; $P<0.01$ in both), while the level of NO was negatively correlated with PASP $(r=-0.696, P<0.01)$. There was also a significantly negative correlation between the serum levels of Rho kinase and ET-1 and the serum level of NO $(r=-0.522,-0.592$, respectively; $P<$ 
0.01 in both). These results suggest that Rho kinase may play an important role in the pathogenesis of $\mathrm{PH}$ in pedriatric patients with CHD. As important active mediators in the regulation of vascular tension, ET-1 and NO may be involved in the formation of $\mathrm{PH}$; this conclusion is consistent with relevant reports [11-14].

In conclusion, serum expression levels of Rho kinase were higher in pediatric patients with left-to-right shunt CHD and were positively correlated with PASP. The expression of serum Rho kinase was even higher in patients with $\mathrm{PH}$. Thus, our results suggest that Rho kinase may interact with ET-1 and NO and participate in the occurrence and development of $\mathrm{PH}$ in pediatric patients with $\mathrm{CHD}$. It is, therefore, of great clinical importance to measure the levels of Rho kinase, ET-1, and NO in pediatric patients with CHD, since these measurements can be used as a reference index to determine the degree of $\mathrm{PH}$, and may provide a theoretical basis for the targeted treatment of children with left-to-right shunt $\mathrm{CHD}$ accompanied by $\mathrm{PH}$.

\section{Abbreviations}

\section{ET-1}

endothelin-1

NO

nitric oxide

$\mathrm{CHD}$

congenital heart disease

PASP

pulmonary artery systolic pressure

non-PAH

non-pulmonary artery hypertension

$\mathrm{PH}$

pulmonary hypertension

RAP

right atrial pressure

Angll

angiotensin II

\section{Declarations}

\section{Funding}

This study was funded by the Jiangsu province "333 project" Cultivation Fund Subsidy Project (BRA2015151). The funding body had no role in the design of the study and collection, analysis, and interpretation of data and in writing the manuscript. 


\section{Competing interests}

The authors declare that they have no competing interests.

\section{Acknowledgements}

We would like to acknowledge the hard and dedicated work of all the staff that implemented the intervention and evaluation components of the study.

\section{Ethics approval and consent to participate}

I confirm that I have read the Editorial Policy pages. This study was conducted with approval from the Ethics Committee of Huai'an First People's Hospital.This study was conducted in accordance with the declaration of Helsinki.Written informed consent was obtained from all participants.

\section{Consent for publication}

All patient guardians signed a document ofinformed consent.

\section{Availability of data and materials}

We declared that materials described in the manuscript, including all relevant raw data, will be freely available to any scientist wishing to use them for non-commercial purposes, without breaching participant confidentiality.

\section{Authors' contributions}

SXZ and HJ conceived the idea and conceptualised the study. TXY and HZ collected the data. SXZ analysed the data. SXZ and TXY drafted the manuscript, then $\mathrm{HJ}$ and $\mathrm{HZ}$ reviewed the manuscript. All authors read and approved the final draft.

\section{References}

1. Chopra S, Badyal DK, Baby PC, Cherian D. Pulmonary arterial hypertension: Advances in pathophysiology and management. Indian J Pharmacol. 2012;44(1):4-11.

2. Rosenkranz S, Ghofrani HA, Beghetti M, Ivy D, Frey R, Fritsch A, Weimann G, Saleh S, Apitz C. Riociguat for pulmonary arterial hypertension associated with congenital heart disease. Heart. 2015;101(22):1792-9.

3. Sun XZ, Tian XY, Wang DW, Li J. Effects of fasudil on hypoxic pulmonary hypertension and pulmonary vascular remodelingin rats.Eur Rev Med Pharmacol Sci,2014,18(7):959-64.

4. Alvira CM, Sukovich DJ, Lyu SC, Cornfield DN. Rho kinase modulates postnatal adaptation of the pulmonary circulation through separate effects on pulmonary artery endothelial and smooth muscle 
cells. Am J Physiol Lung Cell Mol Physiol.2010, 299(6): L872-L878.

5. Gupta V, Gupta N, Shaik IH, Mehvar R, McMurtry IF, Oka M, Nozik-Grayck E, Komatsu M, Ahsan F. Liposomal Fasudil, a Rho-Kinase Inhibitor, for Prolonged Pulmonary Preferential Vasodilation in Pulmonary Arterial Hypertension. J Control Release. 2013;167(2):189-99.

6. Yücel M, Alp H, Yorulmaz A, Karaarslan S, Baysal T. Prediction of the development of pulmonary arterial hypertension with Tei Index in congenital heart diseases with left-to-right shunt. Turk Kardiyol Dern Ars,2019,47(6):466-475.

7. Hou Y, Sun DD, Yuan LJ, Zhu XY, Shang FJ, Hou CJ, Duan YY. Clinical Application of Superior Vena Cava Spectra in Evaluation of PulmonaryHypertension: A Comparative Echo- cardiography and Catheterization Study. Ultrasound Med Biol. 2016;42(1):110-7.

8. Kasahara S. Congenital heart disease having left to right shunts combined with pulmonary hypertension. Kyobu Geka. 2012;65(8):660-4.

9. Calderón-Colmenero J, Sandoval Zárate J, Beltrán Gámez M. Pulmonary hypertension associated with congenital heart disease and Eisenmenger syndrome. Arch Cardiol Mex. 2015;85(1):32-49.

10. Fukumoto Y. Role of the Rho-kinase pathway in pulmonary arterial hypertension. Nihon Yakurigaku Zasshi,2014,143(4):178-81.

11. Shimokawa H, Sunamura S, Satoh K. RhoA/Rho-Kinase in the Cardiovascular System. Circ Res. 2016;118(2):352-66.

12. Liu CP, Dai ZK, Huang CH, Yeh JL, Wu BN, Wu JR, Chen IJ. Endothelial nitric oxide synthaseenhancing G-protein coupled receptor antagonist inhibitspulmonary artery hypertension by endothelin-1-dependent and endothelin-1-independent pathways in a monocrotaline model. Kaohsiung J Med Sci. 2014;30(6):267-78.

13. Li X, Qiu J, Pan M, Zheng D, Su Y, Wei M, Kong X, Sun W, Zhu J. Correlation between congenital heart disease complicated with pulmonary arteryhypertension and circulating endothelial cells as well as endothelin-1[J].Int J Clin Exp Pathol,2015,8(9):10743-51.

14. Sim JY. Nitric oxide and pulmonary hypertension. Korean J Anesthesiol. 2010;58(1):4-14.

\section{Tables}

Table 1 Comparison of serum levels of Rho kinase, ET-1 and NO in each group

Note: Comparison with control group, ${ }^{\square} P<0.01$; Comparison with the non-PAH

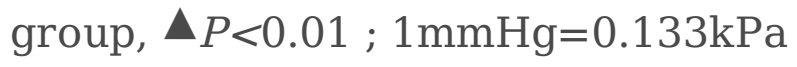




\begin{tabular}{|c|c|c|c|c|c|}
\hline Groups & Cases & Rho kinase & ET-1ロng/L口 & \multicolumn{2}{|c|}{$\mathrm{NO}(\mu \mathrm{mol} / \mathrm{L}) \quad \mathrm{PASP}(\mathrm{mmHg})$} \\
\hline $\begin{array}{l}\text { Control } \\
\text { group }\end{array}$ & 30 & $18.90 \pm 3.07$ & $44.61 \pm 3.88$ & $69.01 \pm 8.40$ & $18.42 \pm 3.68$ \\
\hline $\begin{array}{l}\text { The non- } \\
\text { PAH } \\
\text { group }\end{array}$ & 30 & $28.27 \pm 3.30^{\square}$ & $52.93 \pm 6.86^{\square}$ & $60.74 \pm 8.61^{\square}$ & $19.82 \pm 4.34$ \\
\hline $\begin{array}{l}\text { The mild } \\
\text { PAH } \\
\text { group }\end{array}$ & 30 & $36.70 \pm 6.15^{\square}$ & $78.70 \pm 7.03^{\square}$ & $47.43 \pm 5.44^{\square \boldsymbol{\Delta}}$ & $36.13 \pm 3.82^{\square}$ \\
\hline $\begin{array}{l}\text { The } \\
\text { moderate } \\
\text { to severe } \\
\text { PAH } \\
\text { group }\end{array}$ & 30 & $42.98 \pm 6.60^{\square \Delta}$ & $100.52 \pm 9.18^{\square \Delta}$ & $40.23 \pm 7.32^{\square \Delta}$ & $63.63 \pm 4.86^{\square}$ \\
\hline F & & 128.80 & 396.68 & 88.35 & 750.62 \\
\hline $\mathrm{P}$ & & $<0.001$ & $<0.001$ & $<0.001$ & $<0.001$ \\
\hline
\end{tabular}

\section{Figures}
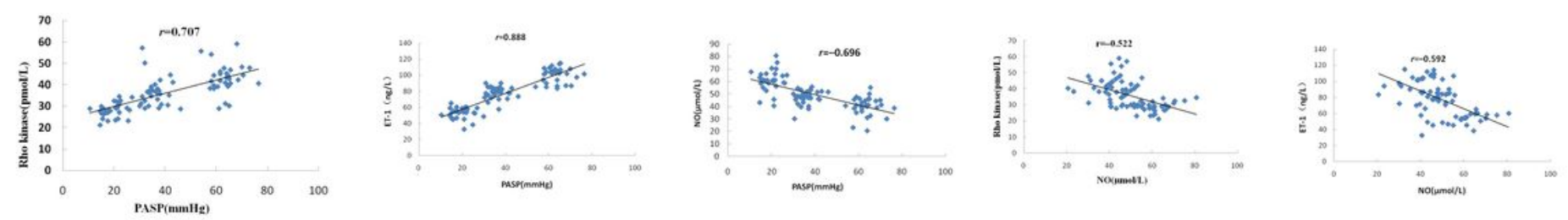

Figure 1

Correlation between the serum levels of Rho kinase, ET-1, NO, and PASP 